\title{
Determining Micromechanical Strain in Nitinol
}

\author{
Matthew Strasberg \\ Office of Science, SULI Program \\ Cornell University \\ Stanford Linear Accelerator Center \\ Stanford, California \\ August 18, 2006
}

Prepared in partial fulfillment of the requirements of the Office of Science, U.S. Department of Energy Science Undergraduate Laboratory Internship (SULI) Program under the direction of Dr. Apurva Mehta in the Stanford Synchrotron Radiation Laboratory (SSRL) at the Stanford Linear Accelerator Center (SLAC).

Participant:

Signature

Research Advisor:

Signature 


\section{Table of Contents}

Abstract

Introduction 1

Materials and Methods $\quad 5$

$\begin{array}{lr}\text { Results and Discussion } & 6\end{array}$

$\begin{array}{ll}\text { Conclusions } & 10\end{array}$

$\begin{array}{ll}\text { Acknowledgements } & 11\end{array}$

$\begin{array}{ll}\text { References } & 11\end{array}$

$\begin{array}{ll}\text { Tables } & 12\end{array}$

$\begin{array}{ll}\text { Figures } & 13\end{array}$ 


\begin{abstract}
Determining Micromechanical Strain in Nitinol. MATTHEW STRASBERG (Cornell University, Ithaca, NY 14853) ERICH OWENS (Albion College, Albion, MI 49224) APURVA MEHTA (Stanford Linear Accelerator Center, Menlo Park, CA 94025) SAMUEL WEBB (Stanford Linear Accelerator Center, Menlo Park, CA 94025)
\end{abstract}

Nitinol is a superelastic alloy made of equal parts nickel and titanium. Due to its unique shape memory properties, nitinol is used to make medical stents, lifesaving devices used to allow blood flow in occluded arteries. Micromechanical models and even nitinol-specific finite element analysis (FEA) software are insufficient for unerringly predicting fatigue and resultant failure. Due to the sensitive nature of its application, a better understanding of nitinol on a granular scale is being pursued through X-ray diffraction techniques at the Stanford Synchrotron Radiation Laboratory (SSRL) at the Stanford Linear Accelerator Center (SLAC). Through analysis of powder diffraction patterns of nitinol under increasing tensile loads, localized strain can be calculated. We compare these results with micromechanical predictions in order to advance nitinol-relevant FEA tools. From this we hope to gain a greater understanding of how nitinol fatigues under multi-axial loads. 


\section{INTRODUCTION}

\section{Nitinol}

A biomedical stent is a lifesaving device used to allow blood flow in an occluded artery. Most stents produced are made of nitinol, shape memory alloy that can be drastically deformed and then heated back to its original shape. This allows for the stent to be compacted for easier insertion into an artery and then expanded, restoring proper blood flow. Once placed in a patient, the stent will expand and contract with each heartbeat. For the typical patient, these stents will undergo some half a billion cycles in the ten year lifetime required by the FDA.

As a result, designing against fatigue is a pressing concern in this industry. Current designs implement micromechanical models and nitinol-specific finite element analysis (FEA) to predict fatigue characteristics. However, stents are failing earlier than anticipated. This deviation can be accounted for by the lack of knowledge of how residual stress accumulates in nitinol. Continuum mechanics, the current model of analyzing stress and strain, is based in the macroscopic scale where elastic and plastic deformations are merely terms to relate the quality of strain in a material. Thus a superelastic material is modeled in the same manner as any other material, just with different numerical constants (Young’s Modulus, Poisson’s ratio, etc...). However, on the granular and atomic scales such deformations have tangible meanings. Atomically, elastic deformation corresponds to a stretching of bonds and plastic deformation refers to breaking of bonds. As a result, understanding the nanocrystalline structure of nitinol is crucial to understanding how loading translates into strain, thus causing fatigue.

\section{Diffraction}

X-ray diffraction can be used to determine nanocrystalline structure utilizing Bragg’s Angle principles and Laue’s equations. Those truly interested in the theory and practical 
derivations should seek an outside text on X-ray diffraction [1], but the basic principles will be discussed in the following. Using a plane-wave source with a wavelength smaller than the lattice spacing, the waves will reflect off the atoms and constructively interfere, Figure 1, if Bragg's equation is satisfied: $2 d \sin (\vartheta)=n \lambda$, where $d$ is the spacing between atomic planes, $\lambda$ is the wavelength, and $2 \theta$ is the supplementary angle between the incident wave and the reflected wave. Meaning the path difference of two waves is equal to some integer multiple of the wavelength. The inverse relation between $d$ and $\theta$ implies the larger the spacing $d$ the smaller the angle of reflection $\theta$. Defining $Q$ as the vector denoting the change in momentum from the incident wave and reflected wave, then $Q=(4 \pi \sin \theta) / \lambda$ and bisects the incident and reflected waves thus pointing along $\mathrm{d}$. Figure 2 is the same geometry, just rotated $\theta$ as a matter of convenience. Note, the incident wave is normal to the sample and is deflected an angle $2 \theta$ as before. As mentioned the change in momentum vector, Q, bisects the angle between the incident wave and the reflected wave or is an angle $\theta$ from the surface plane of the sample. The angle between Q and the surface plane of the sample will be given the generic name $\omega$. This convention is used because for any sample there will be multiple $\mathrm{d}$ spacings and as a result multiple angles of deflection $\theta$, where d spacings are the distance between atomic planes. Thus, $\omega$ is used to just to track the position of the Q vector. Since this is a three dimensional system one more angle is needed to locate $\mathrm{Q}$, that angle will be called $\chi$. Visualize $\chi$ as rotating the $\mathrm{Q}$ vector around the incident beam $\mathrm{I}_{0}$ into the page while maintaining a constant $\omega$ with the surface plane. Physically, $\chi$ corresponds to different orientations of a $\mathrm{d}$ spacing thus the reflected beam can be envisioned as line down the surface of a cone with apex $2 \theta$. In order to extract a diffraction pattern from this configuration, an area detector is placed normal to the incident beam. The detector will intersect the reflected wave cone creating a ring, called a $\chi$ arc or $\chi$ ring, 
in the diffraction pattern, shown in Figure 3. Recalling the inverse relation between $\mathrm{d}$ and $\theta$, the rings closer to the center of the pattern corresponded to the larger $d$ spacings in the atomic structure. Accordingly, there will be a different ring for each d spacing, thus each $\omega$, as shown in Figure 3. In an unstrained case, such as Figure 4, the $\chi$ rings are circular, but as a load is introduced the rings change shape. In order to understand the effect loading has on a diffraction pattern for nitinol, let’s first discuss the micromechanical processes in nitinol.

\section{Nitinol Micromechanics}

There are four micromechanical processes in Nitinol: elastic deformation, phase transformation, twining/de-twining, and plastic deformation. Nitinol has two primary phases in the solid state that are both temperature and strain energy dependent: austenite and martensite. How to create nitinol in a certain phase for a certain temperature is a whole science in itself, but this experiment uses nitinol in the austenite phase at room temperature. Figure 4 is the pattern for the unstrained austenite state, which has concentric circular $\chi$ rings. As a load is introduced, the austenite begins to elastically deform which translates to reversibly stretching of atomic bonds. If the load, thus strain, is uniform in all directions, i.e. hydrostatic, then the $\chi$ rings will maintain their circular shapes but the radius of each ring will change. Due to the inverse relation of $\mathrm{d}$ and $\theta$, if the sample is under compression the radii will increase corresponding to a decrease in d; conversely if the sample is under tension the radii will decrease corresponding to an increase in d. For non-hydrostatic forces, such as the uniaxial tension in this experiment, the $\chi$ rings become elliptical. As the load increases, while still in the range of elastic deformation, the rings become more eccentric. For analysis it is convenient to transform the diffraction pattern from a collection of rings to a series of lines. This is done by unfolding the rings in a program, Fit2D, using the cake function. Figure 5 shows three sample peaks at increasing levels of strain, 
the vertical axis is $\chi$ varying from $-\pi$ to $+\pi$ and the horizontal axis is Q over an arbitrary range in $\mathrm{nm}^{-1}$. The unstrained caked peak is shown in the upper left corner of Figure 5, and is a vertical line. As strain is increased, the peak changes from a straight line to a double arch of increasing eccentricity. As strain increases, the intensity of the peak decreases and starts to disappear. The disappearance of a peak corresponds to the appearance of another peak indicating a phase transformation. Specifically, the austenite is transforming to martensite, which has a different atomic spacing hence the disappearing and appearing of peaks.

The scope of this paper only covers elastic deformation in austenite; however I will discuss the other the micromechanical processes in order to create a broader understanding of diffraction theory. Figure 6 shows nitinol in the martensite phase, the peak is the vertical orange and pink stripe. The variation in intensity within the peak, the purple spots on the first image, relate that there is a certain texture within the material. Although this starting texture was arbitrary, the bright spots do indicate there were more martensite unit cells oriented at $\chi$ zero and $\pi$ in that sample. Again, each successive image in Figure 6 corresponds to an increased level of strain. As strain is increased the texture progresses to a different configuration, one more favorable to the strain conditions. This texture is called the twined state and is recognized by the two distinct bright spots, or poles, on the caked image. Under an even greater strain as shown in the last row of Figure 6, the peak starts to widen and, although not obvious on this scale, starts to lower in intensity. These changes, increase in peak width and decrease in peak intensity, coincide with plastic deformation. Physically, the unit cell clusters are breaking apart shortening the coherence length within the sample. Thus, the intensities of each cluster have a summing effect rather than a multiplicative effect if the sample was still continuous. 
Using diffraction images of austenite under elastic deformation, this paper will discuss and develop techniques for finding strain on the micro-scale. This will be done in Q-space and relate that to the sample geometry. Through analysis of this strain, the effect of macro-strain on the material will become more relevant aiding in design against fatigue.

\section{MATERIALS AND METHODS}

\section{Diffraction}

The diffraction was performed by Apurva Mehta and David Bronfenbrenner at SSRL. The laboratory was setup for routine powder diffraction of a thin strip specimen with a $0.97350 \AA$ beam directly incident on the sample. A thin film of nitinol was placed in a constant strain device and elongated at set increments until 470 microns of elongation. Figure 7 shows the geometry of the strain device inducing; the strain induced by this device will be referred to as

macro-strain. Due to the precision of the strain inducing mechanism, the macro-strain will only be considered in relative terms.

\section{Data processing}

Fit2D, a freeware program http://www.esrf.fr/computing/scientific/FIT2D, was used to convert the raw data into a more usable form. For each pattern, each diffraction ring was isolated and "unfolded" into a linear form using the cake function (see Fit2D help file for information on the cake function). Figure 8 shows three diffraction patterns, three of fourteen in the data set, each at a different level of macro-strain. For each diffraction pattern three $\chi$ rings or peaks (in Miller indices hkl: 110, 200, 211) were caked in Fit2D as shown. These caked images were analyzed in 2DPeakFinder, which extracts the peak position, intensity, and width for every angle $\chi$. 2DPeakFinder is a Matlab script written by [2]. 


\section{RESULTS AND DISCUSSION}

\section{Unstrained $d_{0}$}

The question becomes, at what strain do these micromechanical processes occur? In order to find the strain locally we find the difference in the d spacings along a Q vector and the unstrained $\mathrm{d}$ spacing $\left(\mathrm{d}_{0}\right)$. To find $\mathrm{d}_{0}$ we must analyze the unstrained image. Looking at the caked images, the unstrained peak should be perfectly straight, recall circular ring to straight line and elliptical ring to arches. Practically this is a difficult thing to determine by eye using only the Fit2D images. Thus, a computational tool, a Matlab script, was developed by [2] to analyze the caked image and remove background intensity. This tool assumes a Gaussian shape for peak intensity and thus is able to determine the position of the peak in terms of Q and $\chi$, the width of the peak in Q, and the intensity for each caked image. Figure 9 shows the unstrained 110 peak plotted with Q as a function of $\chi$, which should be a straight line. Since the strain induced is uniaxial, the resulting change in the diffraction ring will be elliptical and thus create symmetric arches in the caked image. However, as shown in Figure 9 there is some variance which is not symmetrical and thus cannot be attributed to strain. It is not expressly stated, but to get the data in the form shown in Figure 9 the data has undergone two filtrations. First, the diffraction pattern was turned into a caked imaged in Q-space using Fit2D. Next, the relevant information from the caked image was extracted in Matlab. Both of these processes introduce some error because of the nature of their algorithms and account for the variance in the data. In order to locate the location of the unstrained peak in Q-space, the data was fit using a basic two element

Fourier model with $\mathrm{Q}$ as a function of $\chi: \quad Q(\chi)=a_{0}+a_{1} \cos f \chi+b_{1} \sin f \chi \quad$ where $a_{0}, a_{1}, b_{1}$, and $f$ are all constants. Giving the unstrained Q location as $\left[a_{0}\right]$ and [the original data $\left.-a_{0}\right]$ as a calibrating factor to adjust for the error discussed above. The calibrating factor for the 110 peak 
will be subtracted from each strained set of data for the 110 peak. This process is repeated for every unstrained peak $(110,200,211)$. Once knowing the location in Q for the unstrained peak, $\mathrm{d}_{0}$ can be found using the relation $d=2 \pi / Q$. This information is shown in Table 1.

\section{Strain Formulae}

Knowing $d_{0}$, the only other information needed to find strain along the Q vector is $d_{x, \omega}$. The strained data undergoes the same filtrations, Fit2D and 2dPeakFinder, thus must utilize the calibration factor mentioned above. For the sake of clarity, we will only discuss the 110 peak and will provide the necessary definitions in Table 2. We will refer to $\mathrm{Q}_{\mathrm{fit}}$ as the desired result for which all strain calculations will be done. $\mathrm{Q}_{\mathrm{fit}}$ is the result of a weighted Fourier fit $\left(Q_{f i t}(\chi)=a_{0}+a_{1} \cos f \chi+b_{1} \sin f \chi\right)$. Using this type of fit assumes the deviation from the unstrained image is due to purely axial loading. The input of the Fourier fit is ( $\mathrm{Q}_{\text {strain }}$ - Calibrant) as a function of $\chi$ and refined by weighting each point by its Intensity. As discussed before, the $\mathrm{Q}$ vector, or specifically $\mathrm{Q}_{\mathrm{fit}}$, can be located using angles $\chi$ and $\omega$. Thus the strain is given as: $\varepsilon_{\chi, \omega}^{\prime}=\frac{d_{\chi, \omega}-d_{o}}{d_{o}}$, where $d_{\chi, \omega}=2 \pi / Q_{\text {fit }}$ and, since $\omega$ is the same as $\theta$ in this geometry $\omega=\sin ^{-1}\left(\frac{Q_{f i t} \lambda}{4 \pi}\right)$, where $\lambda$ is the wavelength of the incident X-ray. The strain along the $Q$ vector can also be expressed in terms of the sample coordinate system, shown in Figure 10. Logically working backwards, if we assume the strain in the sample coordinate system is of the form of a second order, symmetric tensor having six unique elements then we can transform the sample strain into strain along Q. Accordingly, the strain along the Q vector will be:

$$
\varepsilon_{\chi, \omega}^{\prime}=a_{3 k} a_{3 j} \varepsilon_{j k} \quad a_{i j}=\left|\begin{array}{ccc}
\cos \omega & -\sin \omega \sin \chi & -\sin \omega \cos \chi \\
-\sin \chi \sin \omega & \cos \chi & -\sin \chi \cos \omega \\
\sin \omega & \sin \chi \cos \omega & \cos \omega \cos \chi
\end{array}\right|
$$


Where $a_{i j}$ is the transformation matrix between the two coordinate systems. Giving the strain

$$
\text { along Q as } \quad \begin{aligned}
\varepsilon_{\chi, \omega}^{\prime}= & \left(\sin ^{2} \omega\right) \varepsilon_{x x}+(\sin \chi \sin 2 \omega) \varepsilon_{x y}+(\cos \chi \sin 2 \omega) \varepsilon_{x z} \\
& +\left(\cos ^{2} \omega \sin ^{2} \chi\right) \varepsilon_{y y}+\left(\cos ^{2} \omega \sin 2 \chi\right) \varepsilon_{y z}+\left(\cos ^{2} \omega \cos ^{2} \chi\right) \varepsilon_{z z}
\end{aligned}
$$

Knowing both sides of the equation for various $\chi$ and $\omega$, the strain matrix can be determined. Rather than solving an absurdly large system of equations, instead the method of least squares can be applied modeling this system as the sum of six independent variables, each term in parenthesis, with corresponding coefficients, each accompanying strain term. This equation is different than one discussed by [3], which was derived by geometric means. We believe the discrepancy is due to a mathematical error in [3].

\section{Strain Calculations}

Specifically, this least squares routine was run for three $\chi$ rings $(110,200,211$ peaks) for $\chi$ ranging from $-\pi$ to $+\pi$. The fit was performed for fourteen levels of macrostrain, but we will discuss in detail the relative strain case 350. Figure 11a,b, and c plot the the 110, 200, and 211 peaks with the calibrated data in red and the fitted data in blue. Looking at Figure 11c, there is a dip in the data values around $\chi=1.5$. This dip is due to the force apparatus blocking the $\mathrm{X}$-ray from hitting the dector which resulted in a gap in the caked image. The Fourier fitting process adjusts for this. Processing these peaks through the least squares routine mentioned above results in the strain tensor and estimated standard deviations (esds), which are shown in Table 3. Looking at the strain tensor, the axial strain in the y direction corresponds to the direction of the exhibited force and, accordingly, is positve and larger in magnitude than the strain in the other directions. The strains in the orthogonal directions are negative and smaller in magnitude as expected. Figure 12 shows microstrain, in percent strain, versus relative macrostrain, unitless. The shear strains are consistently zero, as mandated by the Fourier fit, thus demonstrating the 
validity of this process of finding strain. In addition, the strains in the principle axes increase in magnitude proportionally with marcostrain. However, the strain in the $\mathrm{z}$ direction is larger in magnitude than the strain in the $\mathrm{x}$ direction. This indicates an anisotropy in the material, either a property of the microstructure or of the strain geometry. Figures 13a-f show show each strain element plotted with their $95 \%$ cofidence intervals. Strain the y and z directions have very small esds indicating a very high level of percision. On the other hand, strain in the x-direction has much larger esds indicating that more data is needed to decrease this noise. To elaborate, the $\mathrm{x}$ direction is perpendicular to the sample surface and the displacement in that direction is a function of $\omega,(d \cos \omega)$. While d varies inversely to $\mathrm{Q}$, the relation between $\mathrm{Q}$ and $\omega$ is given by: $\omega=\sin ^{-1}\left(\frac{Q \lambda}{4 \pi}\right)$. Even with a large deviation in $Q$ space, say from 41.5 to $42 \mathrm{~nm}^{-1}$ for the 200 peak, cos $\omega$ changes from 0.9469 to $0.9455(-0.13 \%)$. Thus, strain in the $\mathrm{x}$ direction is being defered from effectively only three points, one from each $\chi$ ring, while the strain in the $y$ and $\mathrm{z}$ directions is obtained from a much larger population.

\section{Hydrostatic Strain}

Recall the fitting format $Q_{f i t}(\chi)=a_{0}+a_{1} \cos f \chi+b_{1} \sin f \chi$. The $a_{0}$ term refers to a position in Q that can be considered the position of the peak in the caked image. Some $d_{\text {strain }}$ can be determined for every $a_{0}$, (a value in q space), at every level of stain and ultimately translated into hydrostatic strain along the Q vector. Hydrostatic strain refers to axial strain uniform in all directions. Figure 14, shows hydrostatic strain plotted relative to macro-strain for each $\chi$ ring. Notice the hydrostatic strain of 200 peak increases at a much faster rate than the other peaks. This is may be an indication of needing to recalibrate the diffraction image in Fit2D for every strain level. Otherwise, strain is dependent on $h k l$, the peak number. In other words, it 
reconfirms the presence of anisotropy in the material which may become more obvious for higher orders of strain.

\section{CONCLUSION}

The strain matrices developed from this method were qualitatively accurate. The microstrains shown in Figure 12 responded to the macro-strains as expected. Furthermore, the strain equations and method of implementation were valid. Further quantitative analysis revealed a directional dependence for strain in the sample. This was found both in the difference between strain in the $\mathrm{x}$ and $\mathrm{z}$ direction as well as the $h k l$ dependence in the hydrostatic strain. Looking at the Fourier fit, the deviation between the original data and the fitted data appears to increase with strain, shown in Figure 15. However, this deviation, although small, is not symmetric about $\chi$. The Fourier fit used only has a single sine and cosine which fits the data to be symmetric with respect to $\chi$, which is fine for a uniaxial case such as in this paper. However, if a shear strain was introduced that would result in an unsymmetrical arch. Thus, either the mechanism used to exert a force on the nitinol sample was not ideal in introducing a pure axially axial force. The Fourier Fit needs to be more complex in order to fit data with shear strain. In addition, future research can: determine how much information from the diffraction image is needed to extract the strain tensor, experiment with other types of loads, and look at higher levels of strain in relation to the other micromechanical properties. In order to increase the precision of the results, future research should run a new calibration for each level of strain and extract more $\chi$ rings per diffraction image as this paper only deals with three of an available five. 


\section{ACKNOWLEDGEMENTS}

This research was conducted at the Stanford Synchrotron Laboratory at the Stanford Linear Accelerator Center. I provide my greatest appreciation to my mentor Apurva Mehta and fellow SULI student Erich Owens. Through them both I have learned multitudes. I thank David Bronfenbrenner for providing me with data. I thank SLAC and the Department of Energy, Office of Science. Lastly, thanks to Stephanie Majewski.

\section{REFERENCES}

[1] B.E. Warren, X-Ray Diffraction, New York: Dover Publications, 1969.

[2] E. Owens, “Development of powder diffraction analysis tools for a nanocrystalline specimen: An emphasis upon NiTi (Nitinol).” Office of Science, SULI Program, Stanford Linear Accelerator Center, Menlo Park, CA. Tech. Rep. August 25, 2006.

[3] M. Bibee, “Mapping Strain in Nanocrystalline Nitinol: an X-ray Diffraction Method.” Office of Science, SULI Program, Stanford Linear Accelerator Center, Menlo Park, CA. Tech. Rep. August 19, 2005.

[4] Mitchell, Michael, Nitinol Devices \& Components, Freemont, CA. 


\begin{tabular}{|c|c|c|}
\hline Peak Number & Location in Q space (unstrained) & $\mathrm{d}_{0}$ \\
\hline 110 & $29.48578921444401 \mathrm{~nm}^{-1}$ & $2.130919834461030 \AA$ \\
\hline 200 & $41.73517701432516 \mathrm{~nm}^{-1}$ & $1.505489075803596 \AA$ \\
\hline 211 & $51.09763355587002 \mathrm{~nm}^{-1}$ & $1.229643110636340 \AA$ \\
\hline
\end{tabular}

Table 1: Peak number, Location in $Q$ space, and corresponding $d$ spacing for each peak extracted from Nitinol diffraction patterns.

$a_{0}=$ same as $\left[a_{0}\right]$ mentioned in text $=29.48578921444401 \mathrm{~nm}^{-1}$

Calibrant $=\left[\right.$ the original data $-a_{0}$ ] or [the unstrained data $-a_{0}$ ] mentioned in text, a vector of positions in Q-space given in $\mathrm{nm}^{-1}$

$\mathrm{d}_{0}=2.130919834461030 \AA$

$\mathrm{Q}_{\text {strain }}=$ data for the strained case, a vector of positions in Q-space given in $\mathrm{nm}^{-1}$

Intensity = intensity of the peak

$\chi=$ angle relating the diffraction pattern to physical space

$\mathrm{Q}_{\mathrm{fit}}=\mathrm{A}$ vector of calibrated positions in Q-space given in $\mathrm{nm}^{-1}$

Table 2: Definitions for the calibrated fit, referred to as Fourier fit.

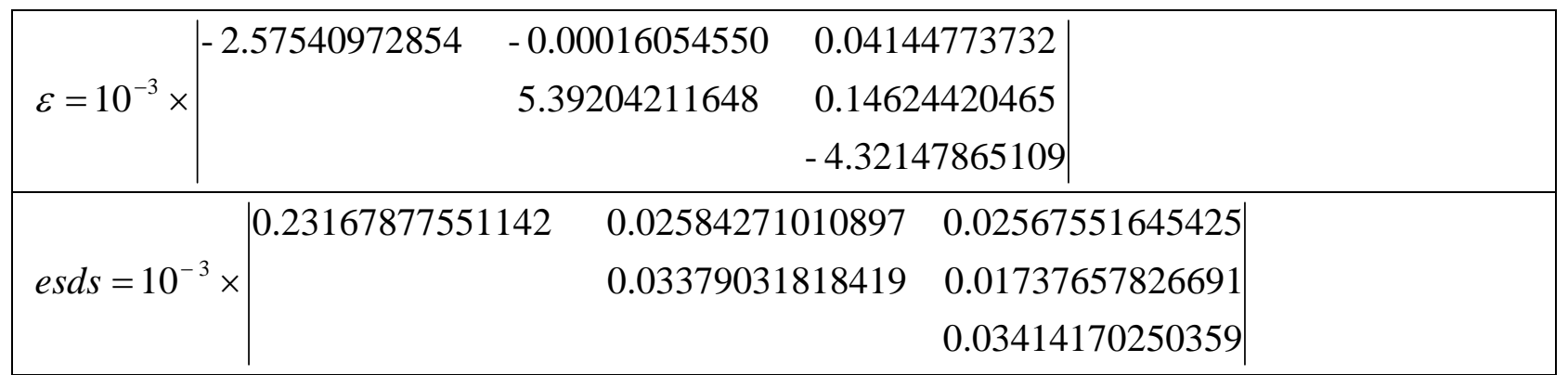

Table 3: Strain tensor and estimated standard deviations for relative macro-strain level of 350. This strain tensor is given in the sample space coordinate system. 


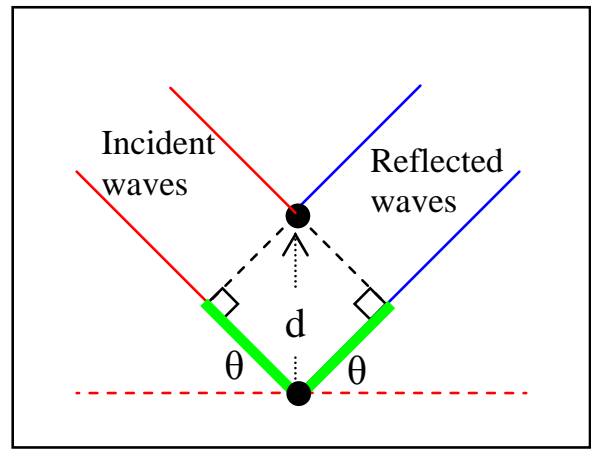

Figure 1: Visualization of Bragg's Law. Incident beam reflects off of atoms by a scattering angle dictated by Bragg's equation.

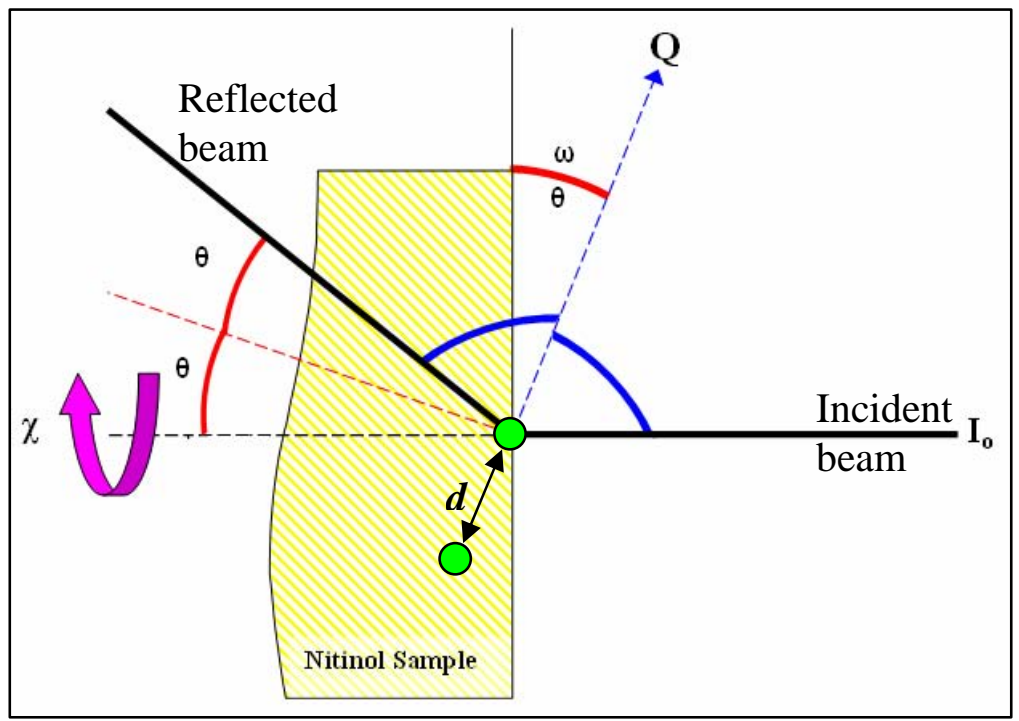

Figure 2: Bragg's Law in transmission geometry. $Q$ vector denotes the change in momentum of the $X$-ray beam $\left(I_{0}\right)$ and points along the $d$ spacing between atoms. Angles $\chi$ and $\omega$ are used to locate $Q$.

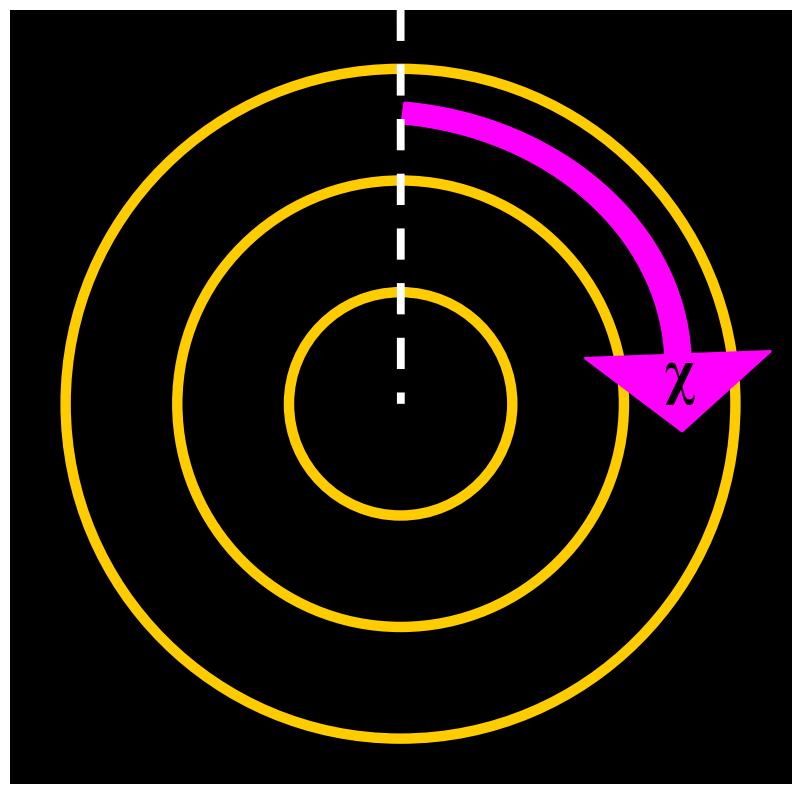

Figure 3: A simplified diffraction pattern. The bright circles are $\chi$ rings, where $\chi$ is the angle from the vertical.

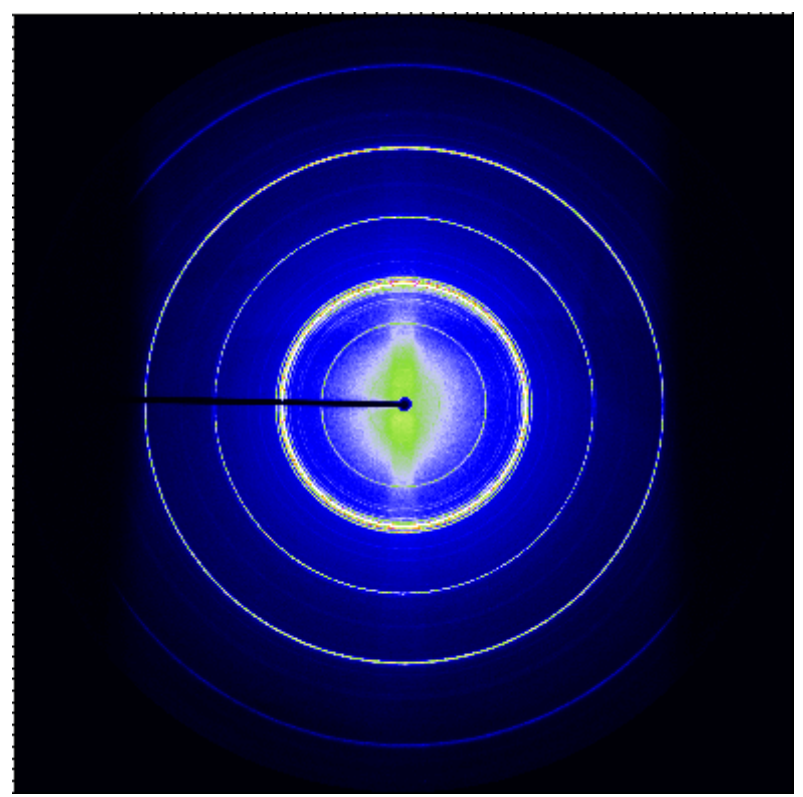

Figure 4: An unstrained diffraction pattern with circular $\chi$ rings. Each ring is referenced by its Miller index, some numbers $h k l$. 

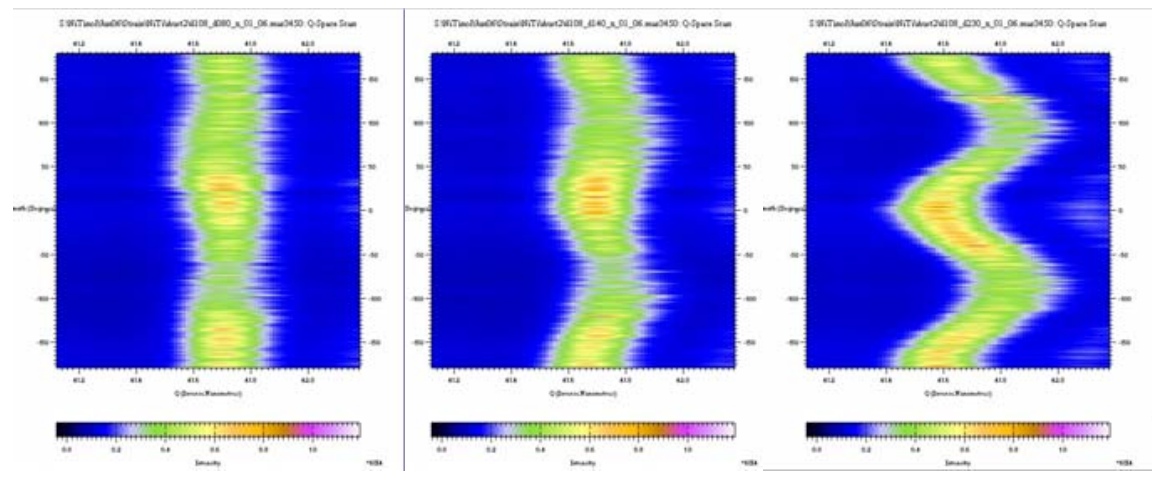

Figure 5: Caked images, straightened $\chi$ rings, of Nitinol in the Austenite phase. Each successive image is under a different level of macro-strain. Notice that the $\chi$ ring progressed from a straight line to an arch of greater eccentricity and eventually begins to disappear. In this process the material is

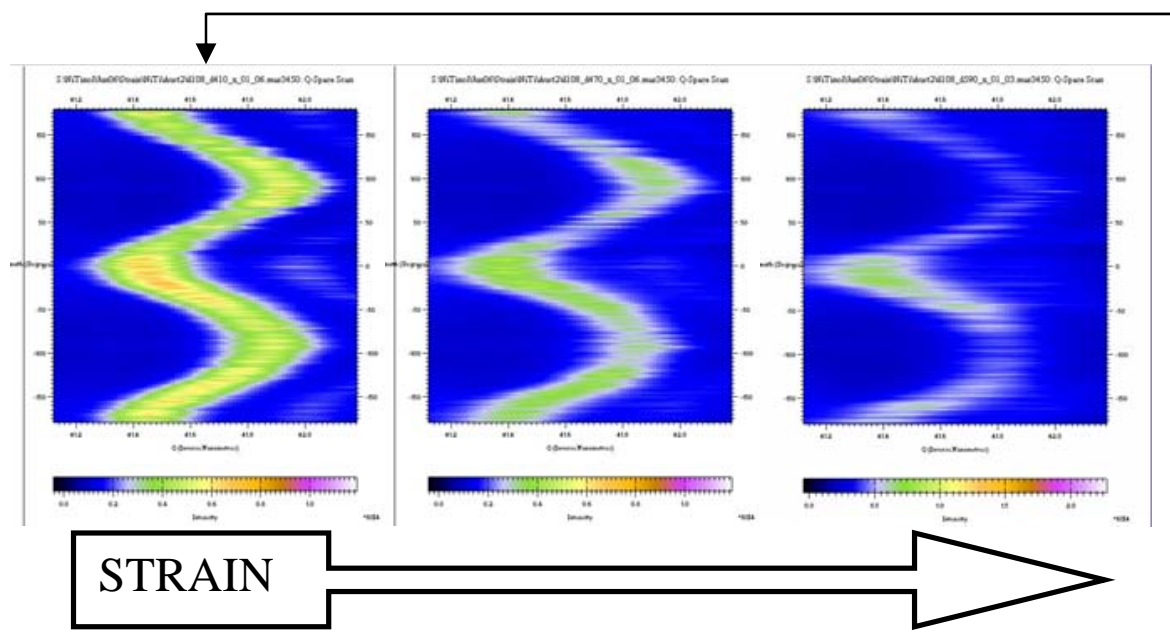
deforming elastically and then undergoing a phase transformation.

Figure 6: Caked images, straightened $\chi$ rings, of nitinol in the martensite phase. Each successive image is under a different level of macro-strain. Notice the change in texture, or twining, as indicated by the intensity poles. As strain increases more, the peaks start to widen, an indication of plastic deformation.
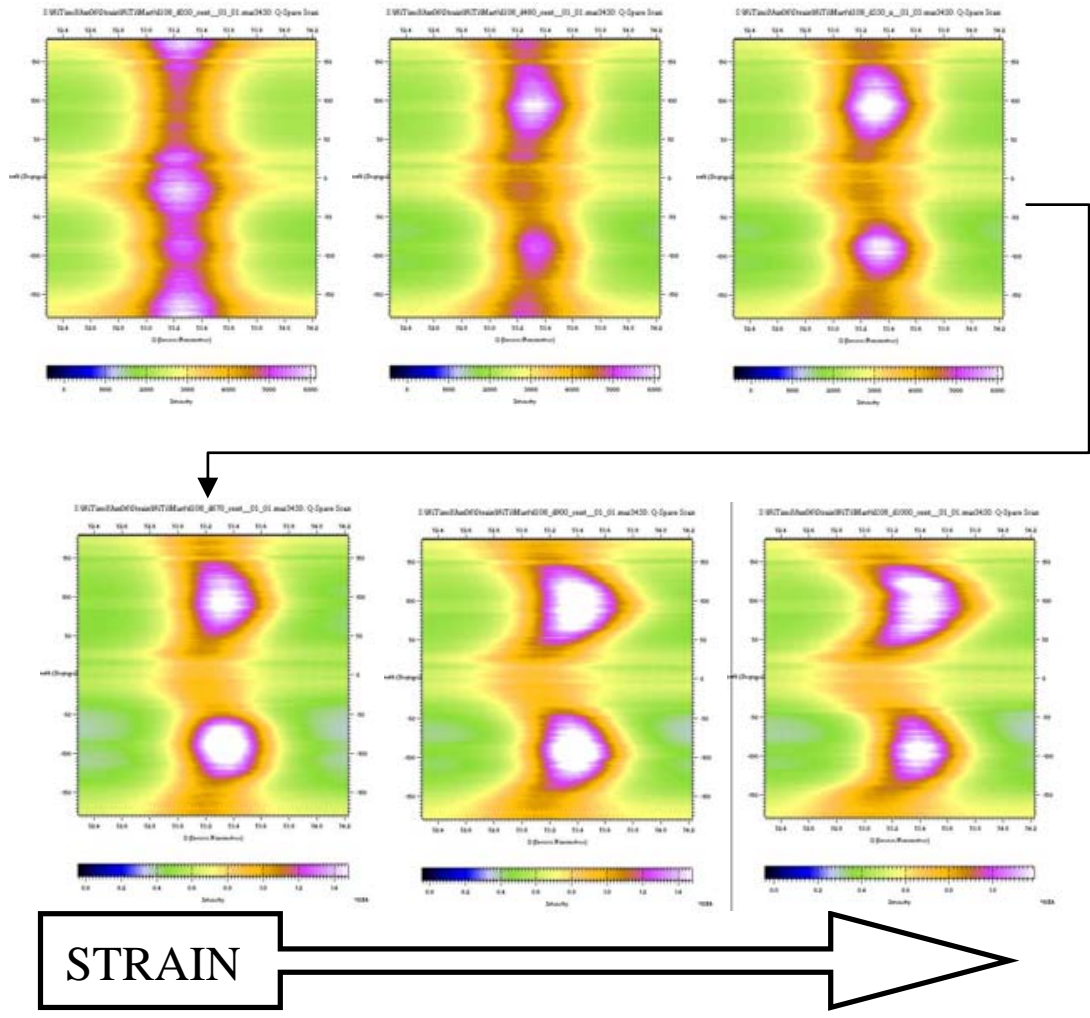


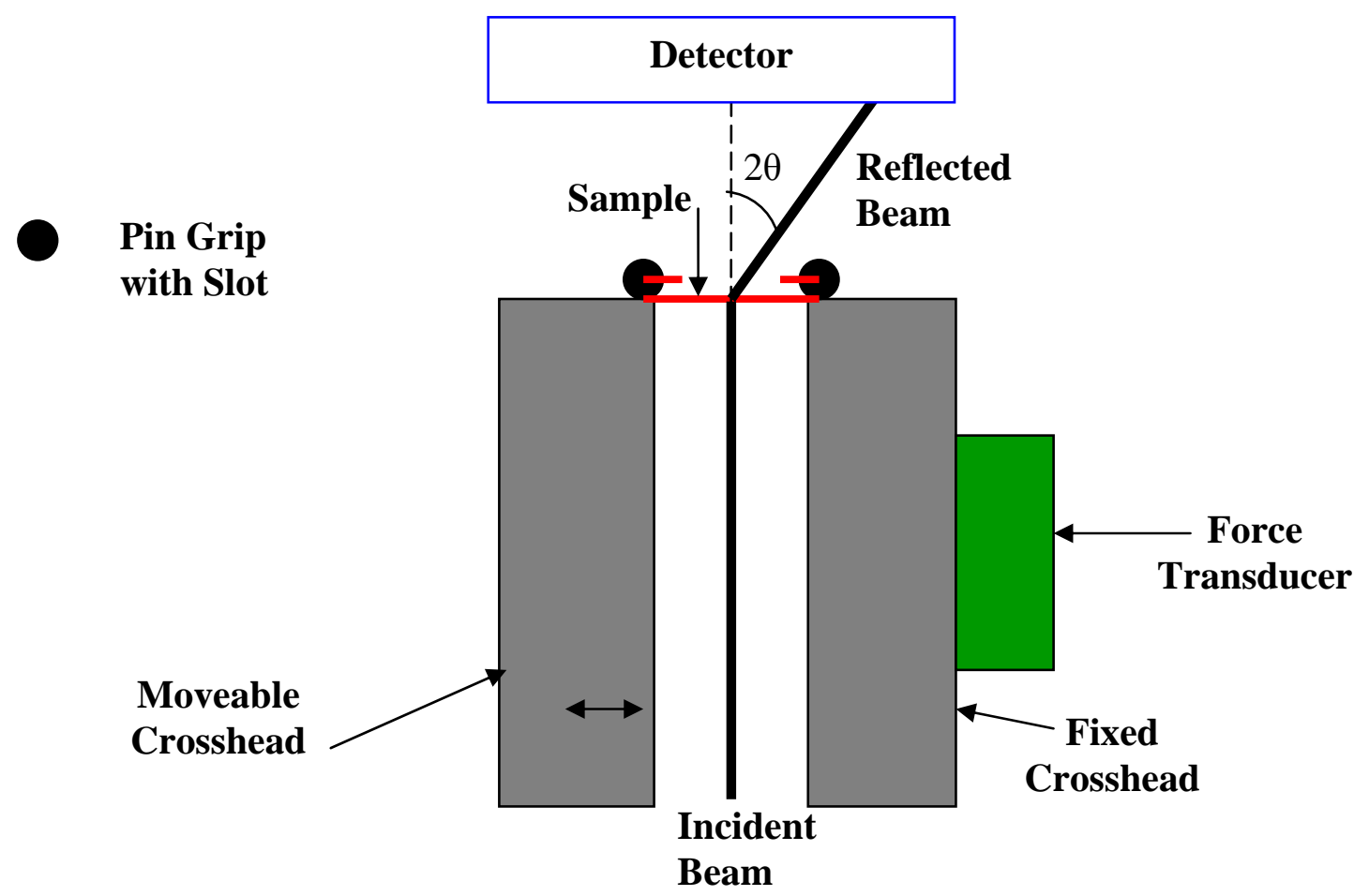

Figure 7: The rig for inducing strain in the sample. The thin film, in red, was pulled out by the moveable crosshead [4]. 


\section{Unstrained}

Relative

Relative

macro-strain of 350

macro-strain of 470

Original diffraction images from the detector. The black trim is a result of shadowing from the force inducing mechanism in Figure 7.

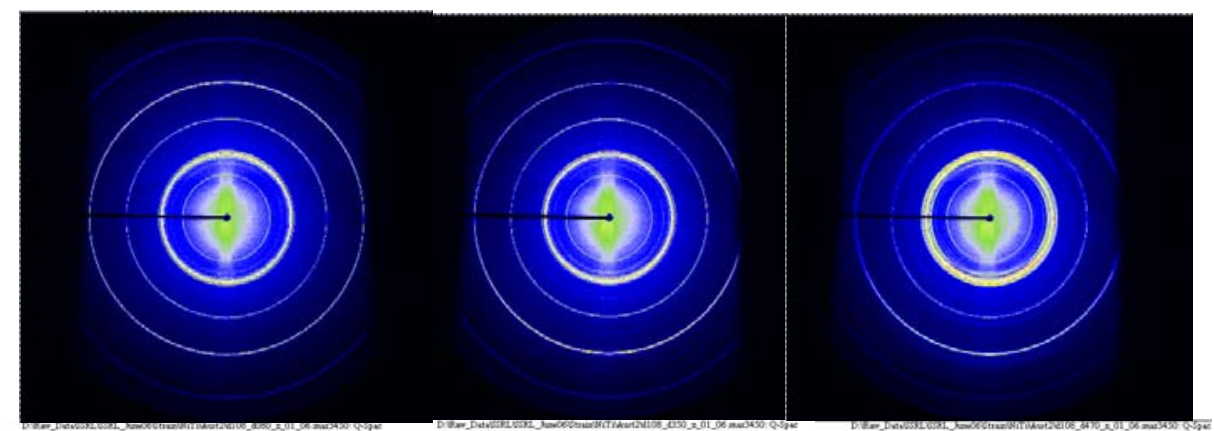

110 Peak (Q29) All shown at same intensity scale
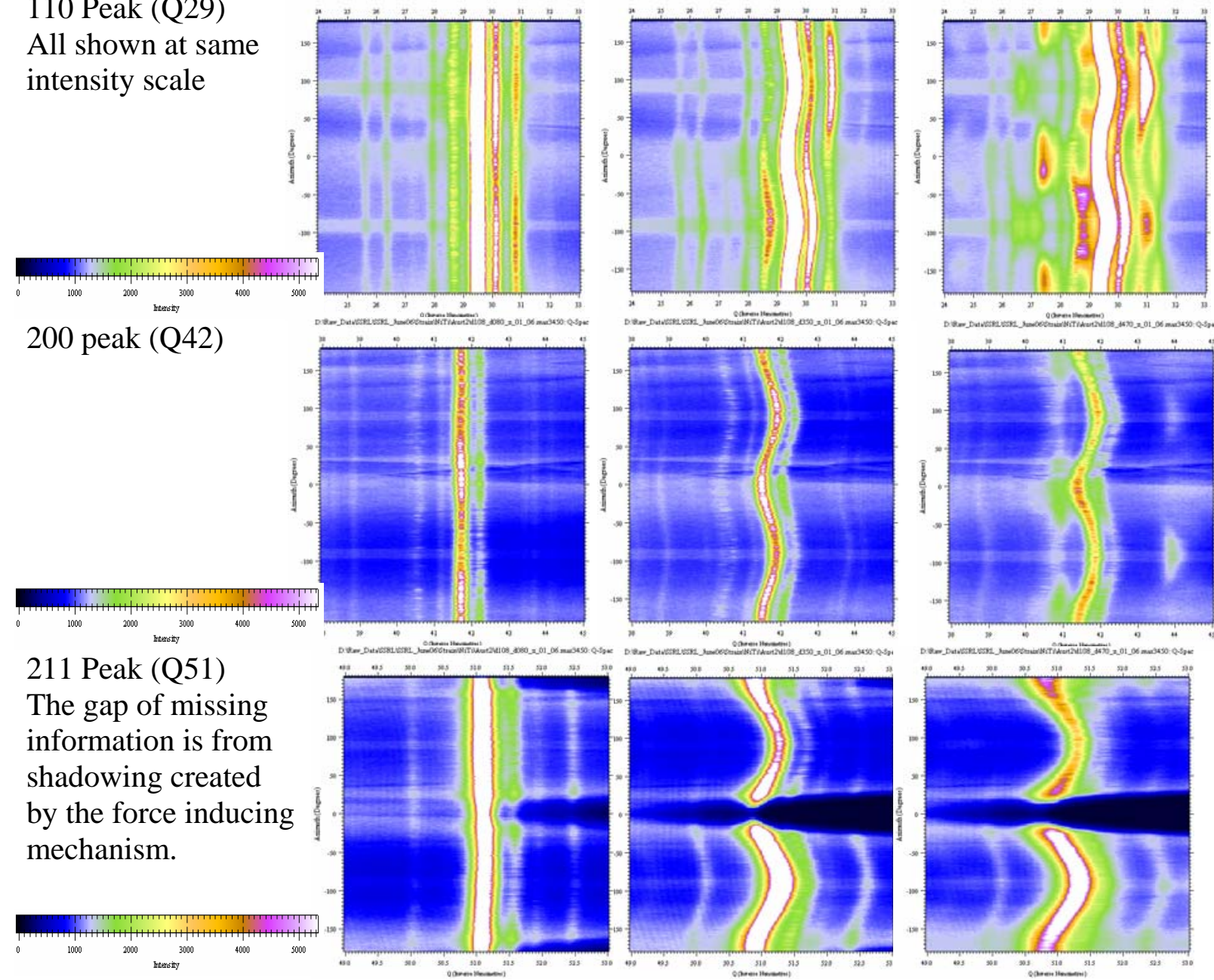

211 Peak (Q51)

The gap of missing information is from shadowing created by the force inducing mechanism.

Figure 8: Each row shows the progression of an intensity peak with strain. Each column shows the different intensity peaks at that level of strain. 


\section{Unstrained 110 Peak}

Location in Q space:

$\mathrm{Q}=29.48578921444401 \mathrm{~nm}^{-1}$

Corresponding $\omega$ :

$\omega=0.23045701838923 \mathrm{rad}$

Unstrained $\mathrm{d}_{0}$ :

$\mathrm{d}_{0}=2.130919834461030 \AA$
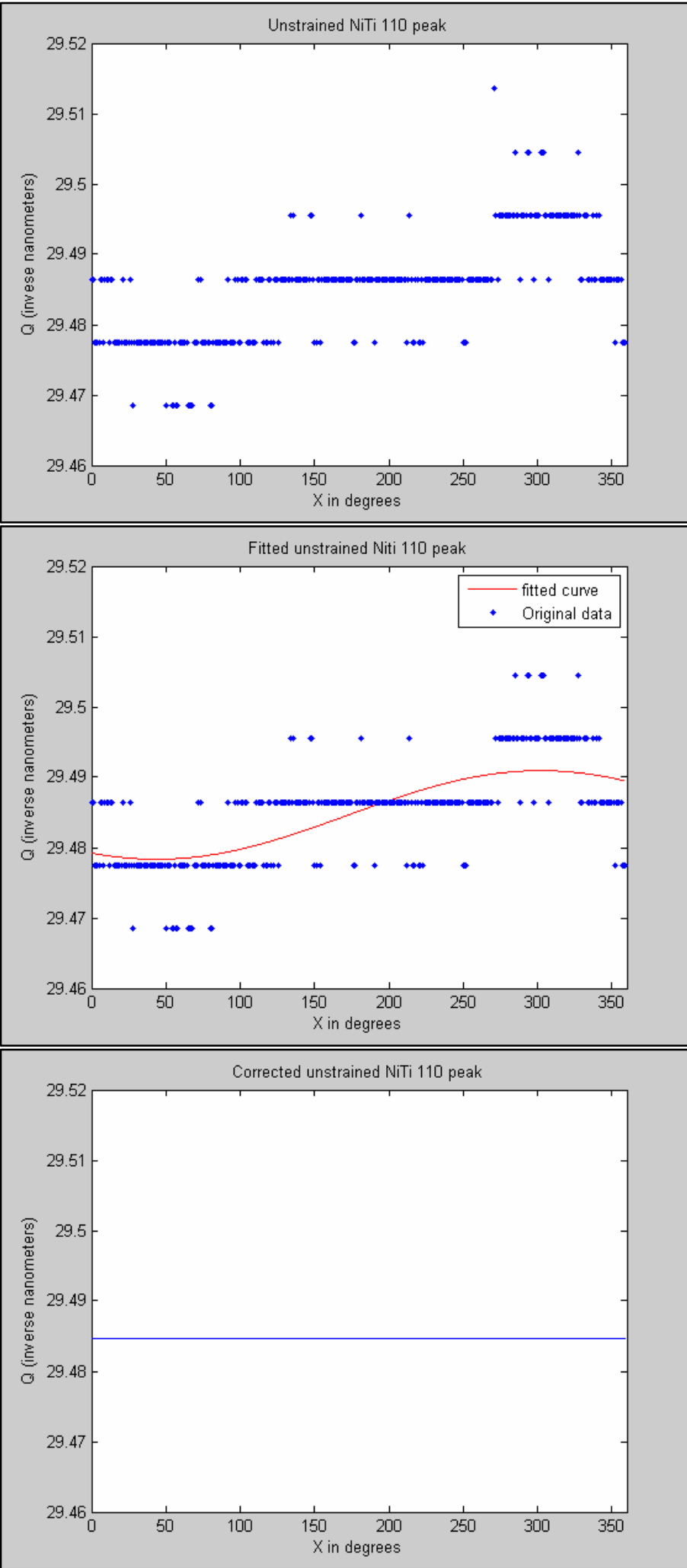

Figure 9: The process of finding $d_{0}$ for the 110 peak. The original data, top, has some unexpected deviations which are attributed to the caking process. Fitting this data allows for a calibration factor to be extracted, which has been applied to the unstrained case, shown at the bottom. 


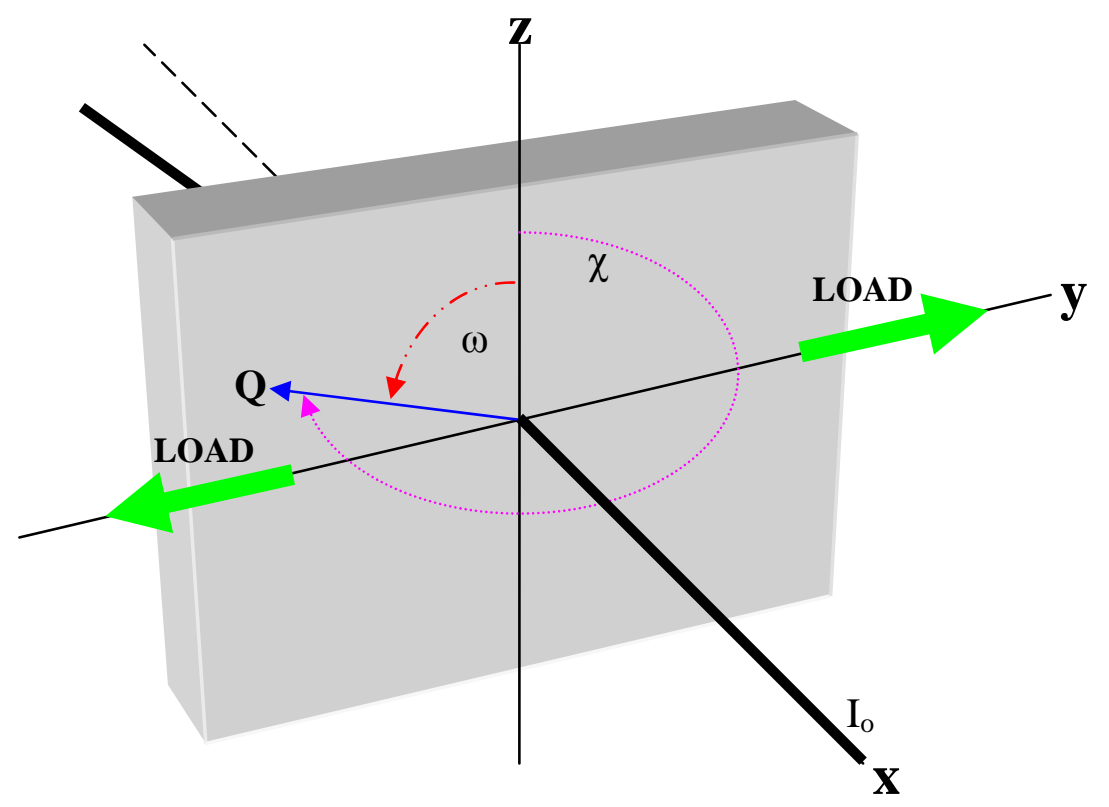

Figure 10: Sample geometry. Angles $\chi$ and $\omega$ are the same from Figure 2 . The force is in the $y$ direction. 

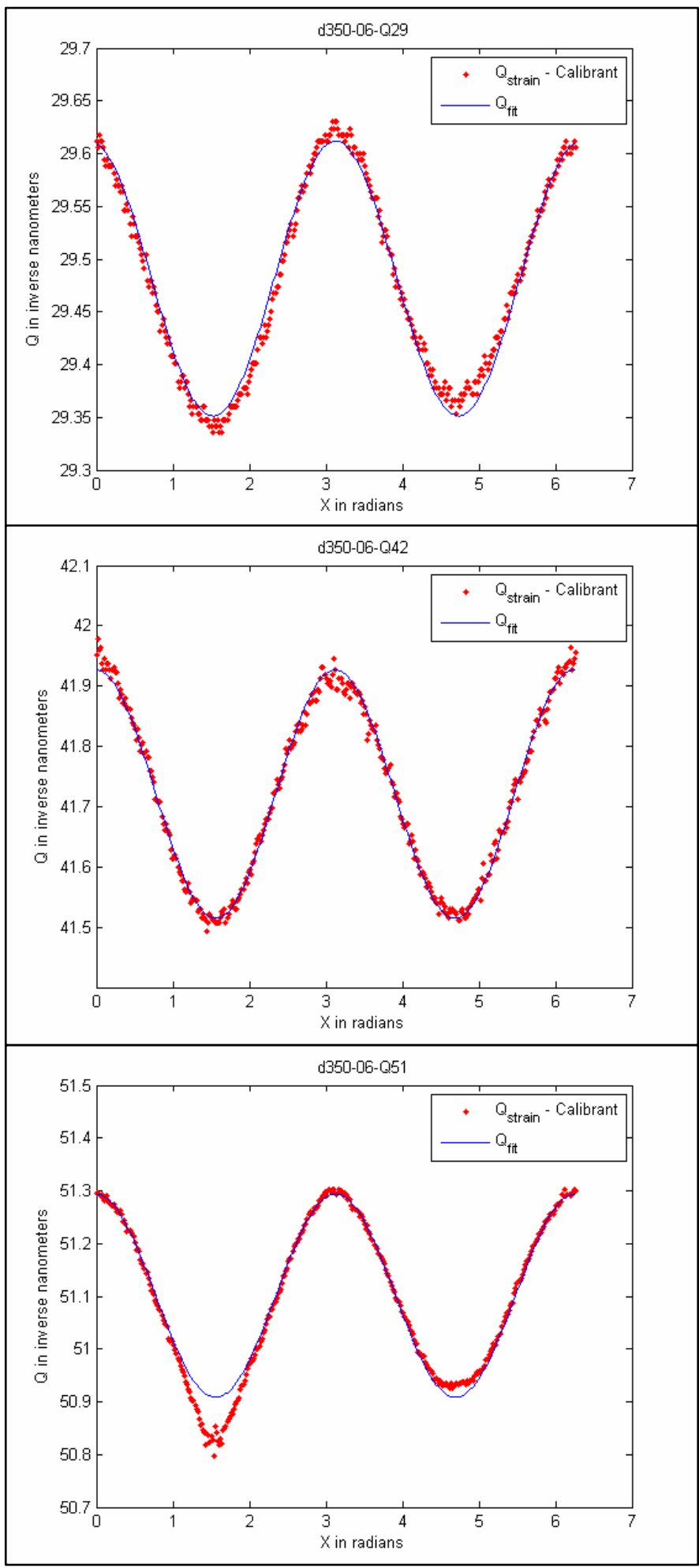

Figure 11: The Fourier fit for each peak (110, 200, and 211) at a relative macro-strain of 350 


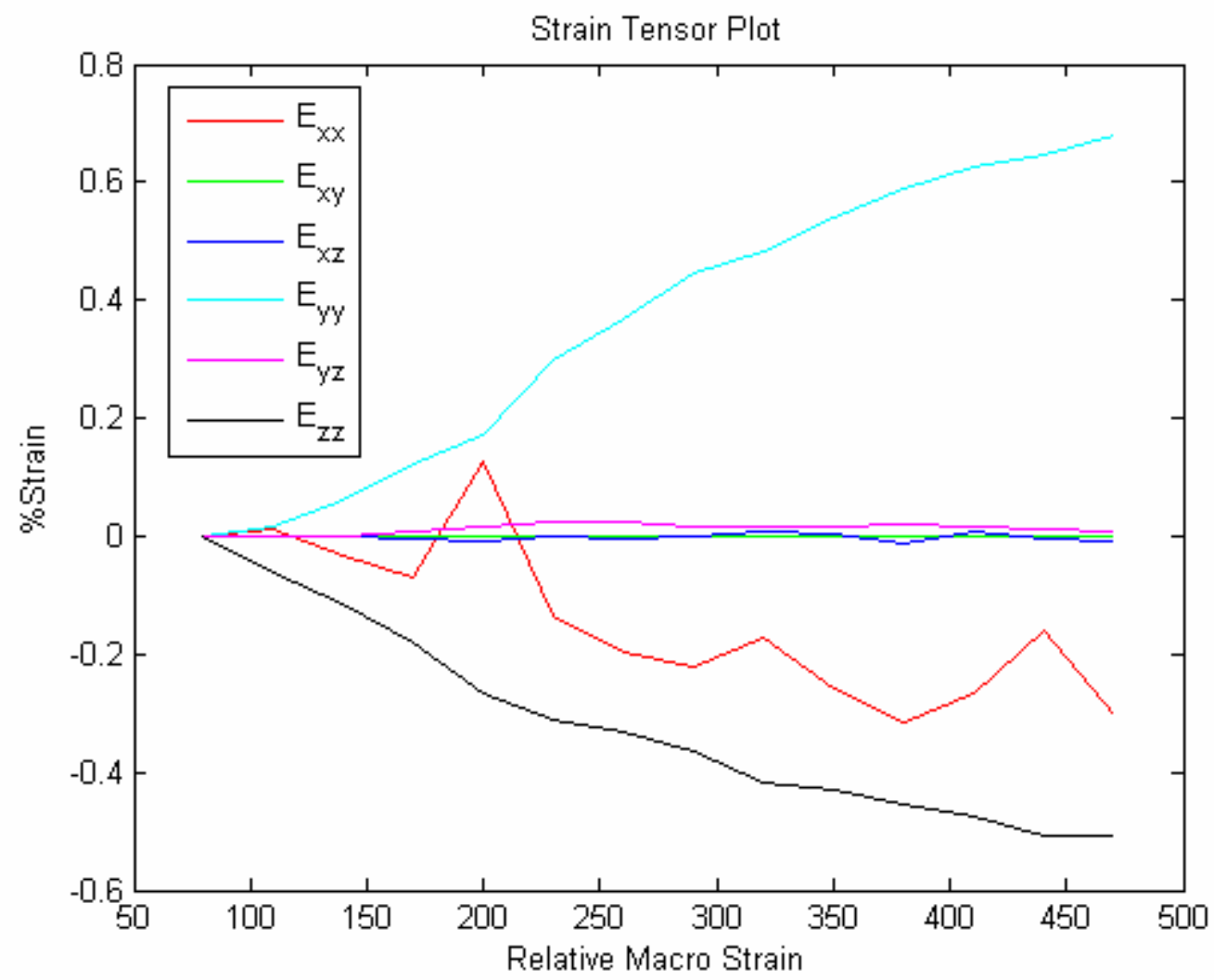

Figure 12: Strain in the direction of load, the cyan line, increases proportionally with the load. Strain in the orthogonal directions is negative and proportional to the load as well. Shear strain is zero for all load levels. 


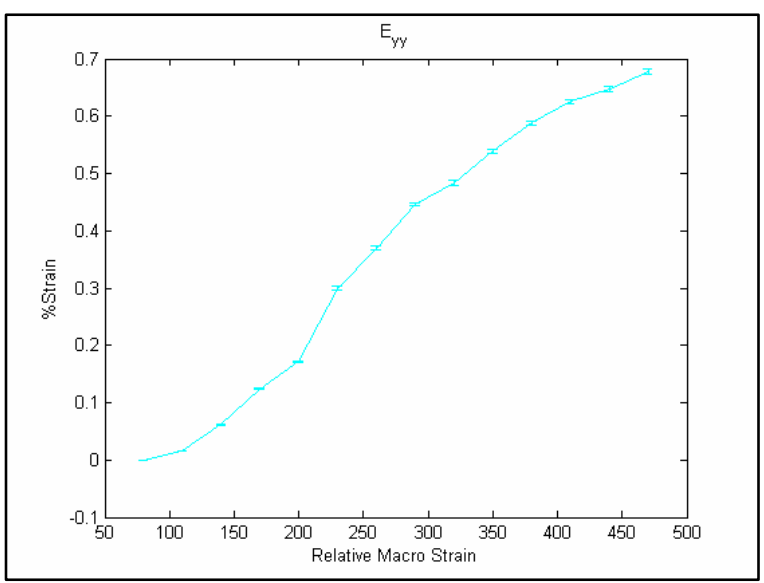

Figure 13.a

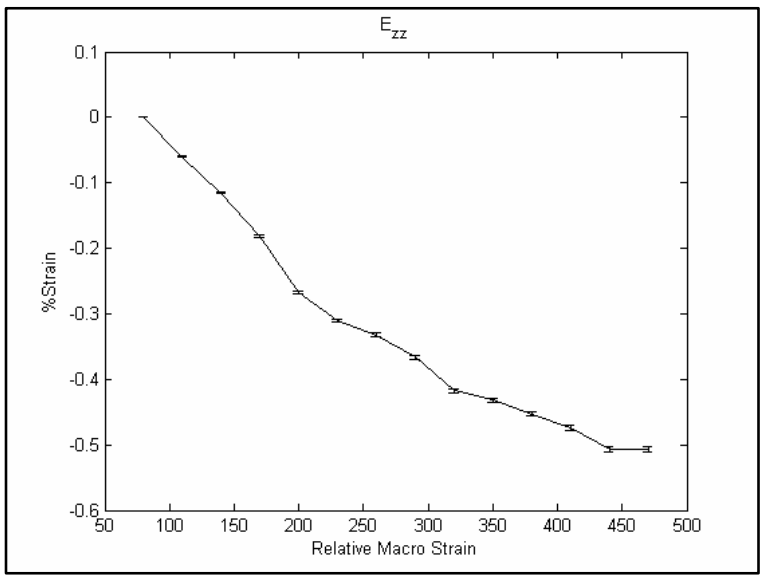

Figure 13.b

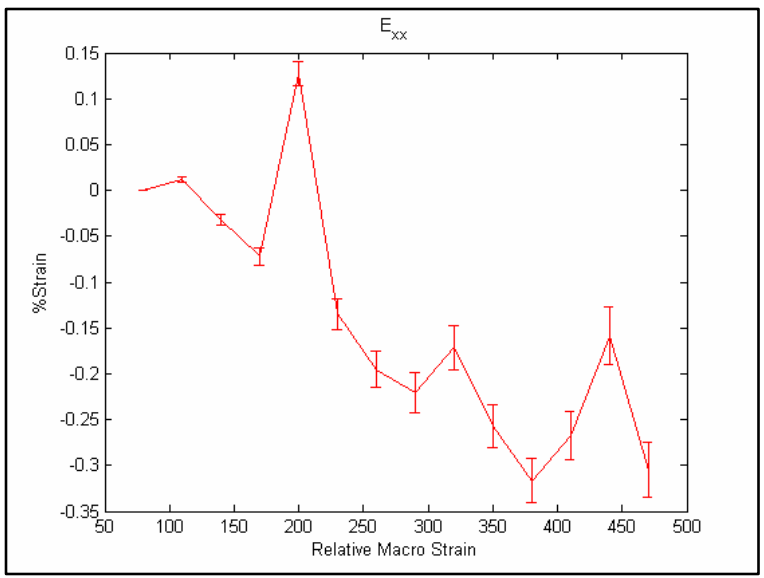

Figure 13.c

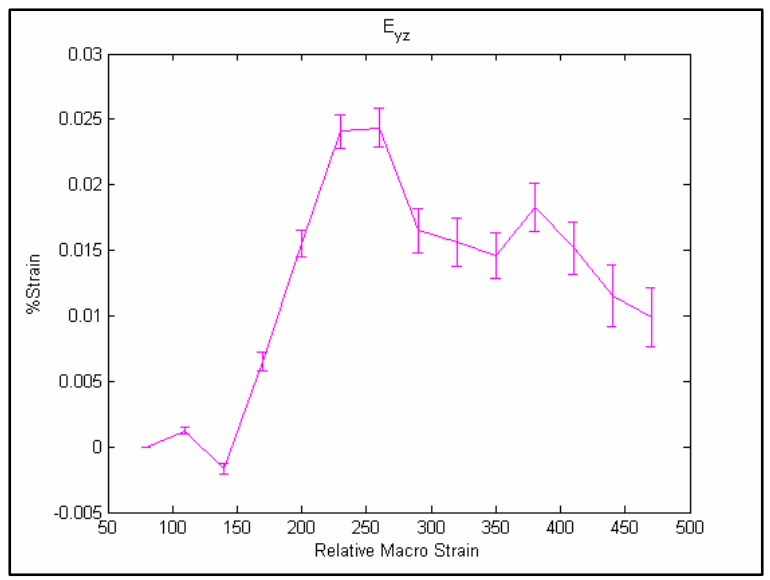

Figure 13.d

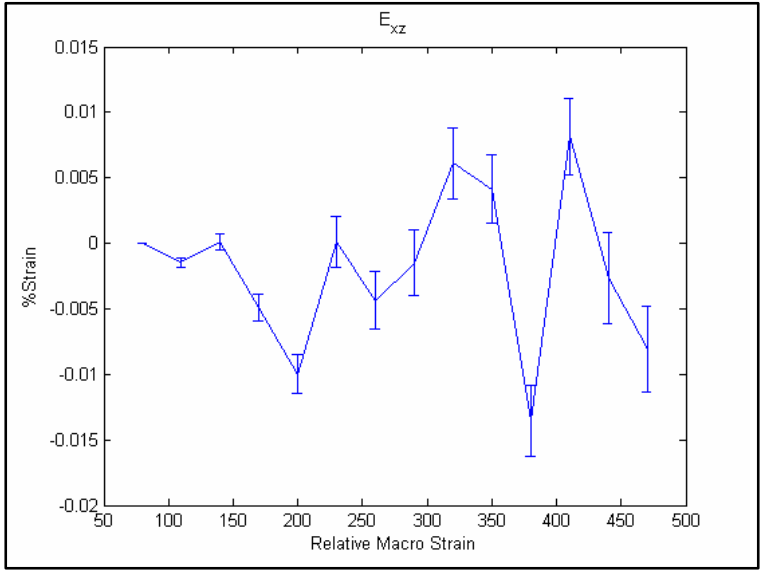

Figure 13.e

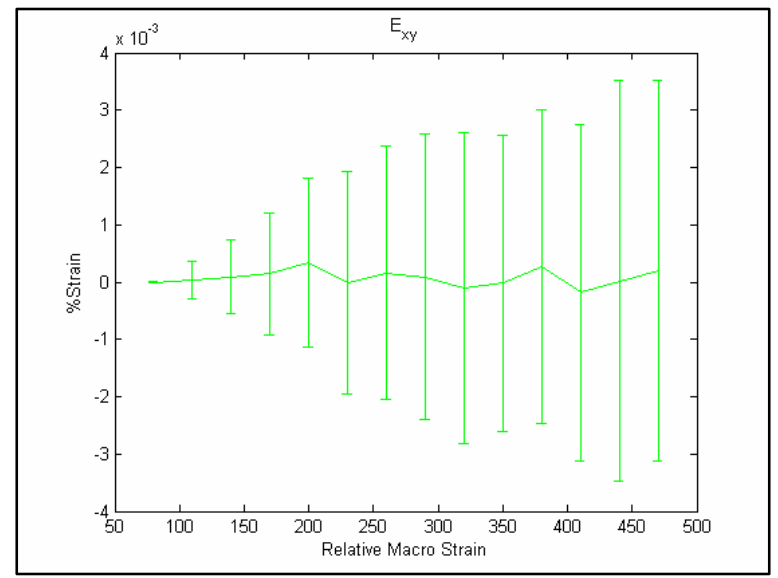

Figure 13.e 


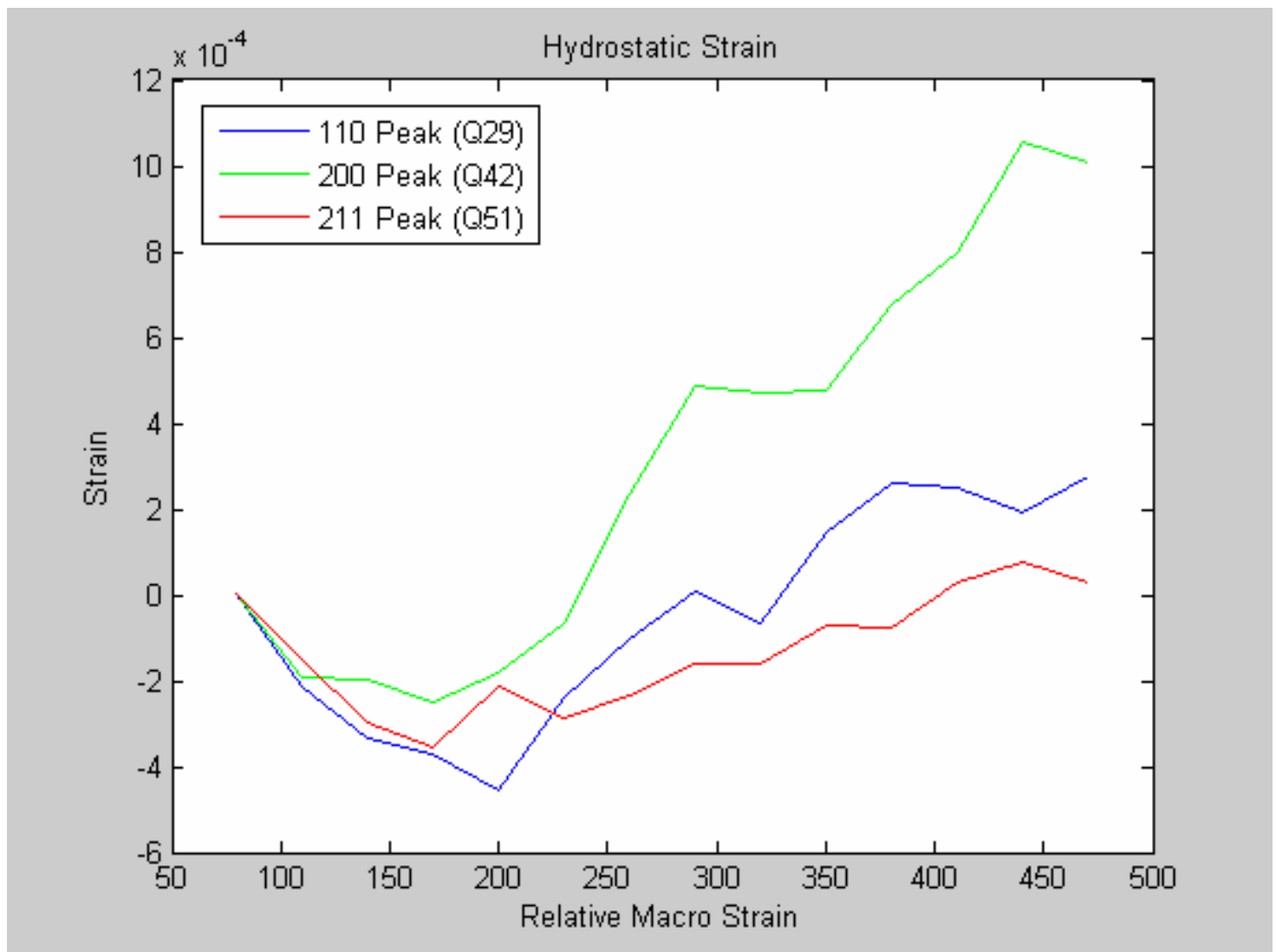

Figure 14: Hydrostatic strain is dependent on $h k l$. 

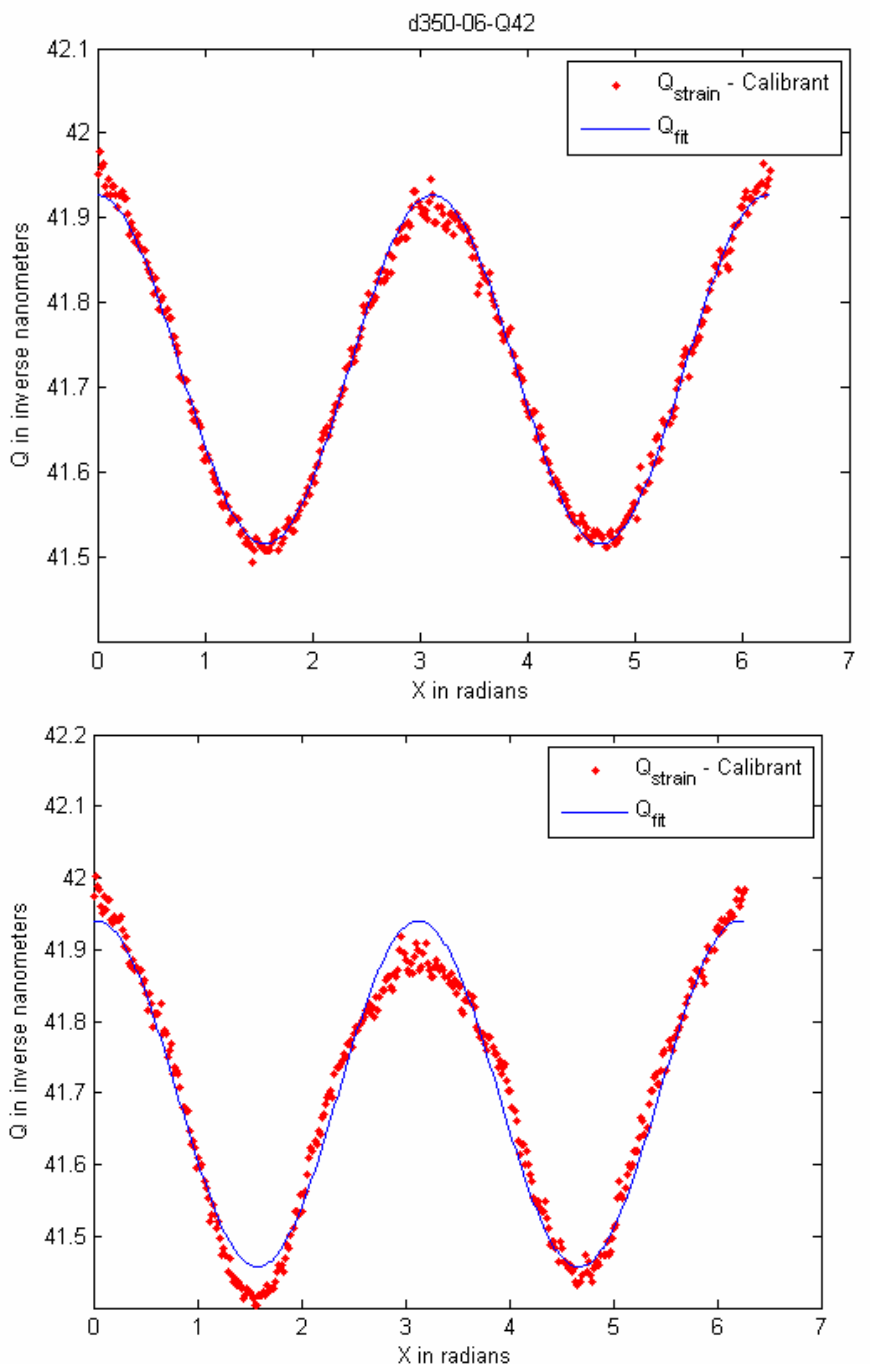

Figure 15: The Fourier fit used to smooth the data we appropriate for small levels of strain, but had trouble fitting the data for higher strain values. 